\title{
Manganese Segregation Behavior in Damascene Metal Lines
}

\author{
Guoda Lian ${ }^{1}$, Malik Ali ${ }^{1}$ and Steve Boettcher ${ }^{1}$ \\ ${ }^{1 .}$ IBM Inc., Hopewell Junction, NY, USA
}

The effective diffusivity in $\mathrm{Cu}$ lines during electromigration (EM) mass flow is related to microstructure and dopant additions and is controlled by fast diffusion paths, such as interfaces between $\mathrm{Cu}$ and $\mathrm{SiCN}$ capping layer, and grain boundaries (GB). The dimensional evolution of on-chip $\mathrm{Cu}$ interconnections from microns to tens of nm-sized line widths has caused a change in $\mathrm{Cu}$ line microstructure, thus resulting in a significant change in diffusion path. Manganese $(\mathrm{Mn})$ as dopant/seed plays a critical role in damascene $\mathrm{Cu}$ lines by paring with $\mathrm{GB}$ vacancies or interfacial oxygen to reduce the diffusivity.

For a decade, $\mathrm{Mn}$ has been added in $\mathrm{Cu}$ seed layers for improving $\mathrm{EM}$ for $\mathrm{Cu}$ lines. Even though the doping level (less than fractions of a percent) is below physical detection limits by analytical transmission electron microscopy (TEM) techniques, the improvement of EM is observed by electrical test [1]. However after thermal stress or reliability tests, Mn segregates to the interfaces and GB at a higher concentration than in bulk $\mathrm{Cu}$ which makes it in the detectable range using an advanced analytical TEM technique on ultrathin lamella prepared with inverted dual beam focus ion beam (FIB). Figure 1 a) is a bright field (BF) image of $\mathrm{Cu}$ metal showing a sharp GB line in the diffraction contrast. Figure $1 \mathrm{~b}$ ) is the corresponding high angle annular dark field (HAADF) scanning transmission electron microscopy (STEM) image, inset red dashed box marks the area where the spectrum image was acquired. Figure $1 \mathrm{c}$ ) and d) are electron energy loss spectroscopy (EELS) maps of Mn and combined EELS maps of Ta ,Cu, Mn, O, and $\mathrm{N}$, in which $\mathrm{Mn}$ segregation is shown at the interface of $\mathrm{Cu}$ and $\mathrm{SiCN}$ capping layer and $\mathrm{Cu} \mathrm{GB}$. In addition, from the fine structures of oxygen $\mathrm{K}$ and $\mathrm{Mn} \mathrm{L}_{2,3}$ edges (such as the chemical shift and the splits of the peak), two different phases of manganese, such as metallic Mn and Mn oxide, can be easily identified. The blue spectrum in Figure 2 b) (taken at $\mathrm{Cu}$ GB) shows only $\mathrm{Mn} \mathrm{L}_{2}$ while lacking $\mathrm{O} \mathrm{K}$ and $\mathrm{Mn} \mathrm{L}_{3}$ peaks indicating the metallic phase appears at the $\mathrm{Cu}$ GB. This may suggest that Mn coupling with the vacancies at $\mathrm{Cu}$ GB, which can slow down GB movement resulted in the improvement of EM reliability. The red spectrum in Figure $2 \mathrm{~b}$ ) (acquired at the interface of $\mathrm{Cu}$ and capping layers) shows both $\mathrm{Mn} \mathrm{L}_{2}$ and $\mathrm{L}_{3}$, which is a clear signature of Mn oxides. Furthermore the pre-edge of oxygen $\mathrm{K}$ and energy split between the $\mathrm{Mn} \mathrm{L}_{2}$ and $\mathrm{L}_{3}$ edge peaks maximum suggests $\mathrm{Mn}_{3} \mathrm{O}_{4}$ appears at the interface, according to reference paper [2]. The existence of $\mathrm{Mn}$ oxides at the interface effectively isolates the $\mathrm{Cu}$ surface from oxygen preventing $\mathrm{Cu}$ oxide formation, which is deleterious to EM reliability.

In conclusion, different Mn segregation behaviors are detected by high resolution STEM/EELS from high quality samples prepared with inverted FIB. Two different phases of Mn segregation are identified with EELS fine structures.

\section{References:}

[1] C.-K. Hu, et al, J. Appl. Phys. 111 (2012), p. 093722.

[2] L. Laffonta, and P. Gibotb, Materials Characterization 61 (2010), p. 1268. 

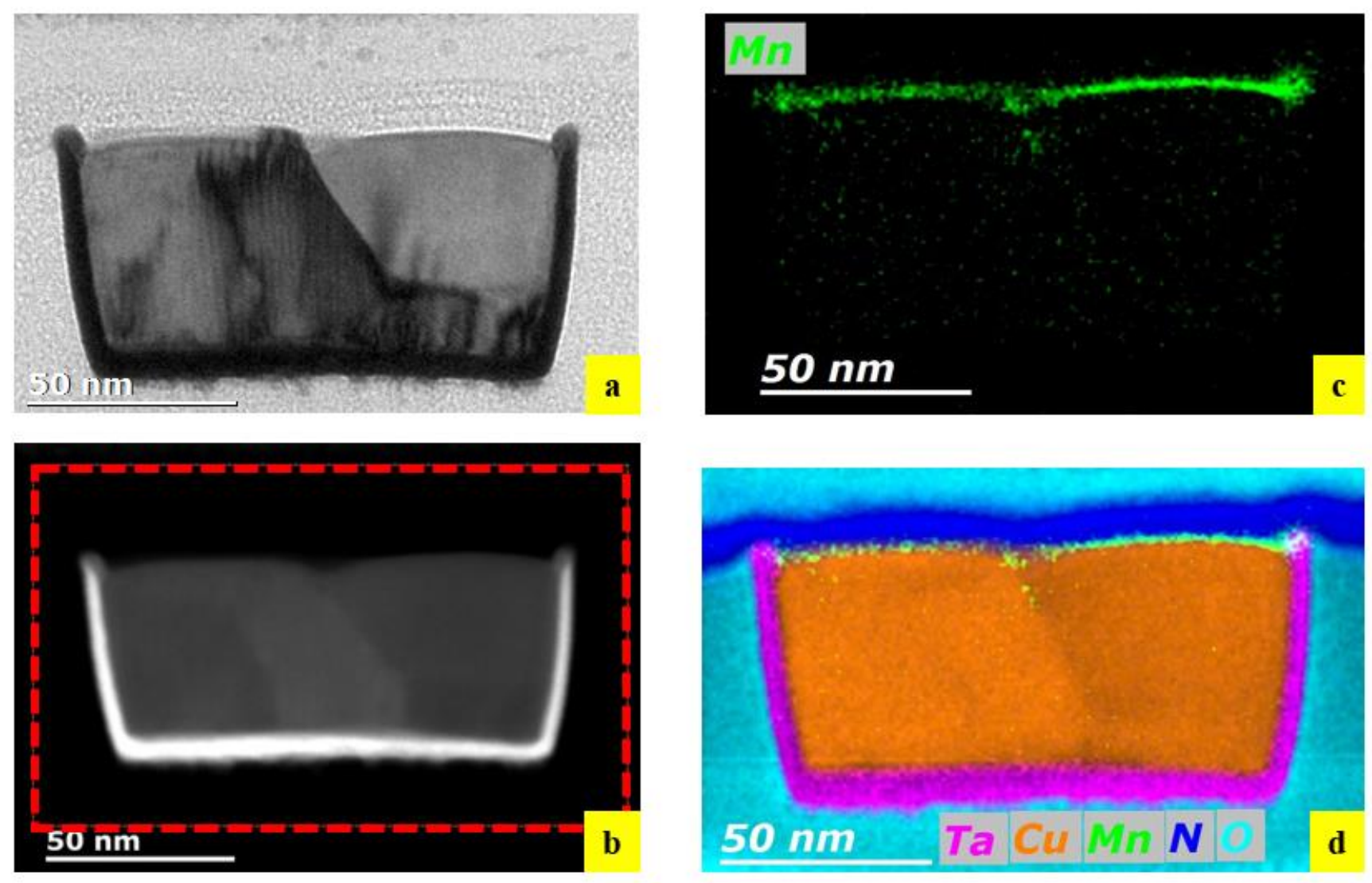

Figure 1. a) Bright field of a typical $\mathrm{Cu}$ line with a GB running through the middle of the metal, b) HAADF image with red rectangle box marking the area where EELS spectrum image was taken, c) Mn EELS map shows the segregation at the GB and the interface, and d) combined EELS maps of Ta ,Cu, $\mathrm{Mn}, \mathrm{O}$, and $\mathrm{N}$.
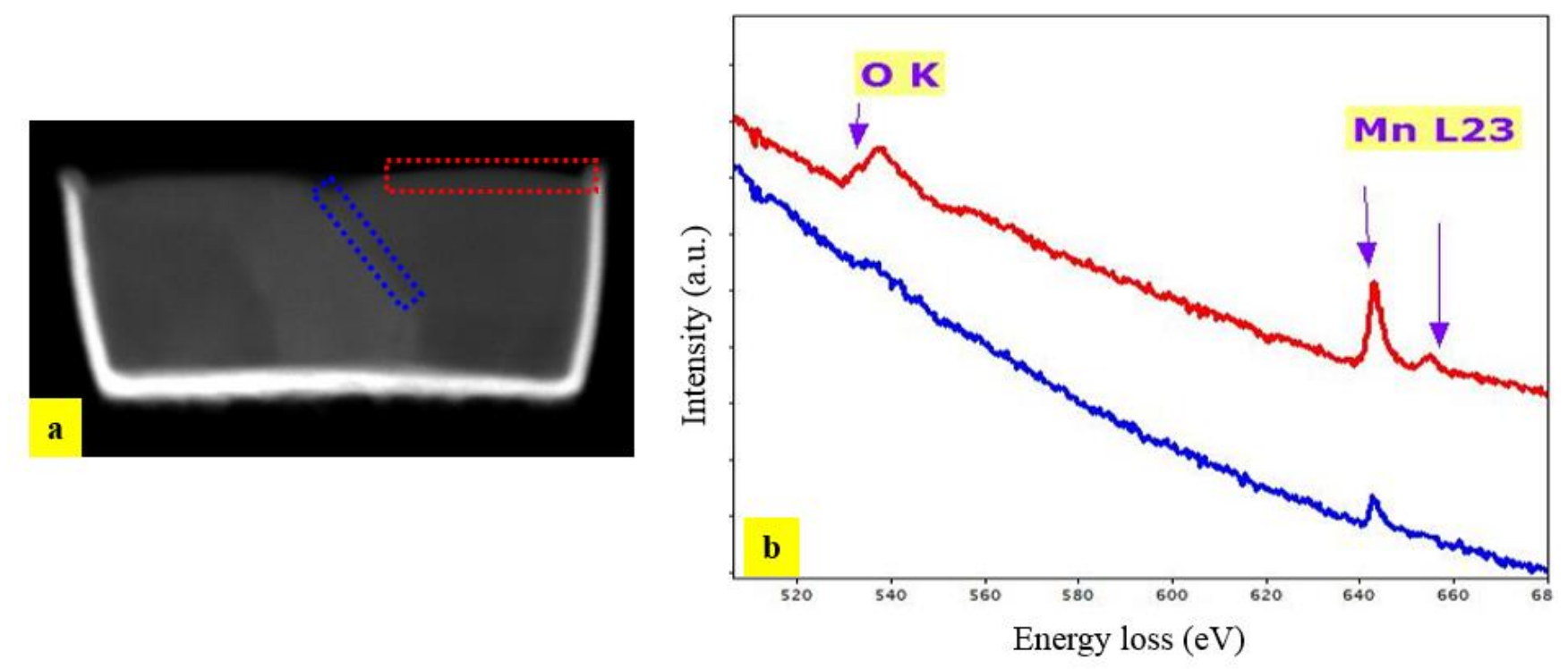

Figure 2. a) HAADF with color boxes showing where the corresponding spectra were acquired, b) EELS spectra taken from GB (blue) and interface (red). 